\title{
Scaling macroscopic aquatic locomotion
}

\author{
Mattia Gazzola', Médéric Argentina ${ }^{2,3}$ and L. Mahadevan ${ }^{1,4 \star}$
}

Inertial aquatic swimmers that use undulatory gaits range in length $L$ from a few millimetres to $\mathbf{3 0}$ metres, across a wide array of biological taxa. Using elementary hydrodynamic arguments, we uncover a unifying mechanistic principle characterizing their locomotion by deriving a scaling relation that links swimming speed $U$ to body kinematics (tail beat amplitude $A$ and frequency $\omega$ ) and fluid properties (kinematic viscosity $v$ ). This principle can be simply couched as the power law $\operatorname{Re} \sim \mathrm{Sw}^{\alpha}$, where $\operatorname{Re}=U L / v \gg 1$ and $\mathrm{Sw}=\omega A L / v$, with $\alpha=4 / 3$ for laminar flows, and $\alpha=1$ for turbulent flows. Existing data from over 1,000 measurements on fish, amphibians, larvae, reptiles, mammals and birds, as well as direct numerical simulations are consistent with our scaling. We interpret our results as the consequence of the convergence of aquatic gaits to the performance limits imposed by hydrodynamics.

Aquatic locomotion entails a complex interplay between the body of the swimmer and the induced flow in the environment ${ }^{1,2}$. It is driven by motor activity and controlled by sensory feedback in organisms ranging from bacteria to blue whales ${ }^{3}$. Locomotion at low Reynolds numbers $(\operatorname{Re}=U L / v \ll 1)$ is governed by linear hydrodynamics and is consequently analytically tractable, whereas locomotion at high Reynolds numbers $(\mathrm{Re} \gg 1)$ involves nonlinear inertial flows and is less well understood ${ }^{4}$. Although this is the regime that most macroscopic creatures larger than a few millimetres inhabit, as shown in Fig. 1a, the variety of sizes, morphologies and gaits makes it difficult to construct a unifying framework across taxa.

Thus, most studies have tried to take a more limited view by quantifying the problem of swimming in specific situations from experimental, theoretical and computational standpoints $s^{5-7}$. Beginning more than fifty years ago, experimental studies ${ }^{8-12}$ started to quantify the basic kinematic properties associated with swimming in fish, while providing grist for later theoretical models. Perhaps the earliest and still most comprehensive of these studies was performed by Bainbridge ${ }^{8}$, who correlated size and frequency $f=\omega /(2 \pi)$ of several fish via the empirical linear relation $U / L=(3 / 4) f-1$. However, he did not provide a mechanistic rationale based on fundamental physical principles.

The work of Bainbridge served as an impetus for a variety of theoretical models of swimming. The initial focus was on investigating thrust production associated with body motion at high Reynolds numbers, wherein inertial effects dominates viscous forces ${ }^{13,14}$. Later models also accounted for the elastic properties of the body and muscle activity ${ }^{15-18}$. The recent advent of numerical methods coupled with the availability of fast, cheap computational resources has triggered a new generation of direct numerical simulations to accurately resolve the full three-dimensional problem ${ }^{19-24}$. Although these provide detailed descriptions of the forces and flows during swimming, the large computational data sets associated with specific problems obscure the search for a broader perspective.
Inspired by the possibility of an evolutionary convergence of locomotory strategies ultimately limited by hydrodynamics, we bring together the specific and general perspectives associated with swimming using a combination of simple scaling arguments, detailed numerical simulations and a broad comparison with experiments. We start by recalling the basic physical mechanism underlying the inertial motion of a slender swimmer of length $L$, tail beat frequency $\omega$ and amplitude $A$, moving at speed $U$ (Fig. 1b) in a fluid of viscosity $\mu$ and density $\rho$ (kinematic viscosity $v=\mu / \rho)$. At high Reynolds numbers $\operatorname{Re}=U L / v \gg 1$, inertial thrust is generated by the body-induced fluid acceleration, and balanced by the hydrodynamic resistance. We assume that the oscillation amplitude of motion is relatively small compared to the length of the organism, and that its body is slender. This implies that fluid acceleration can be effectively channelled into longitudinal thrust ${ }^{14}$. Furthermore, undulatory motions are considered to be in the plane, so that all quantities are characterized per unit depth.

In an incompressible, irrotational and inviscid flow the mass of fluid set into motion by the deforming body scales as $\rho L^{2}$ per unit depth ${ }^{25}$, assuming that the wavelength associated with the undulatory motions scales with the body length $L$, consistent with experimental and empirical observations. The acceleration of the surrounding fluid scales as $A \omega^{2}$ (Fig. 1c) and therefore the reaction force exerted by the fluid on the swimmer scales as $\rho L^{2} A \omega^{2}$. As the body makes a local angle with the direction of motion that scales as $A / L$, this leads to the effective thrust $\rho \omega^{2} A^{2} L$, as shown in Fig. 1c.

The viscous resistance to motion (skin drag) per unit depth scales as $\mu U L / \delta$, where $\delta$ is the thickness of the boundary layer ${ }^{26}$. For fast laminar flows the classical Blasius theory shows $\delta \sim L \mathrm{Re}^{-1 / 2}$, so that the skin drag force due to viscous shear scales as $\rho(v L)^{1 / 2} U^{3 / 2}$, as shown in Fig. 1c. Balancing thrust and skin drag yields the relation $U \sim A^{4 / 3} \omega^{4 / 3} L^{1 / 3} v^{-1 / 3}$ which we may rewrite as

$$
\mathrm{Re} \sim \mathrm{Sw}^{4 / 3}
$$

where $\mathrm{Sw}=\omega A L / v$ is the dimensionless swimming number, which can be understood as a transverse Reynolds number characterizing the undulatory motions that drive swimming. This simple scaling relationship links the locomotory input variables that describe the gait of the swimmer $A, \omega$ via the swimming number Sw to the locomotory output velocity $U$ via the longitudinal Reynolds number Re.

At very high Reynolds numbers $\left(\operatorname{Re}>10^{3}-10^{4}\right)$, the boundary layer around the body becomes turbulent and the pressure drag dominates the skin $\mathrm{drag}^{26}$. The corresponding force scales as $\rho U^{2} L$ per unit depth, which when balanced by the thrust yields

$$
\mathrm{Re} \sim \mathrm{Sw}
$$

\footnotetext{
${ }^{1}$ School of Engineering and Applied Sciences, Harvard University, Cambridge, Massachusetts 02138, USA, ²Université Nice Sophia-Antipolis, Institut non linéaire de Nice, CNRS UMR 7335, 1361 route des lucioles, 06560 Valbonne, France, ${ }^{3}$ Institut Universitaire de France, 103, boulevard Saint-Michel, 75005 Paris, France, ${ }^{4}$ Department of Organismic and Evolutionary Biology, Department of Physics, Wyss Institute for Biologically Inspired Engineering, Kavli Institute for Nanobio Science and Technology, Harvard University, Cambridge, Massachusetts 02138, USA. *e-mail: Im@seas.harvard.edu
} 
a

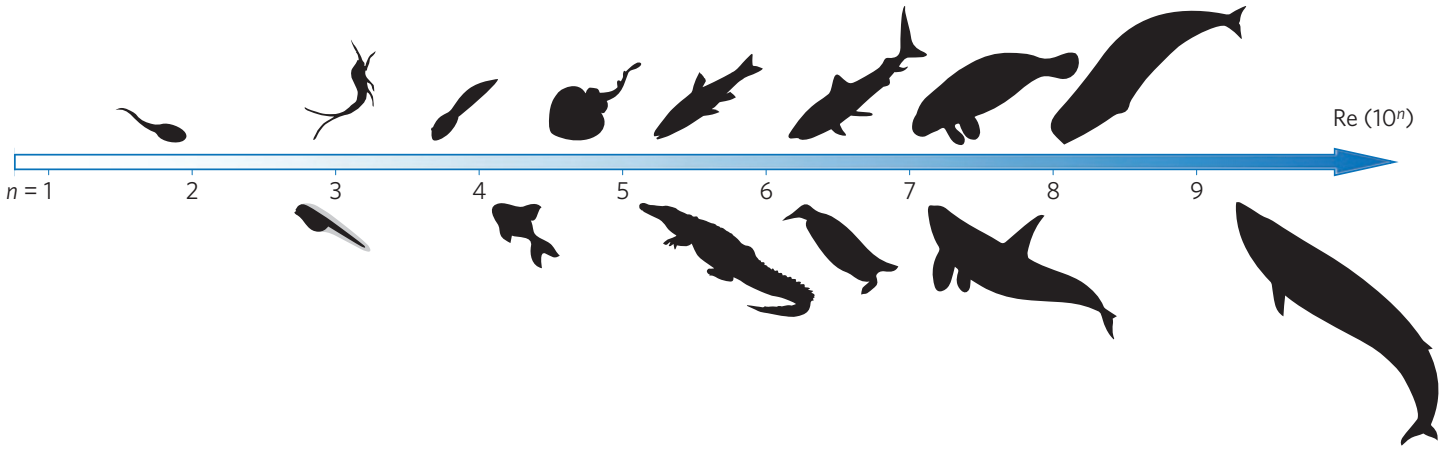

b
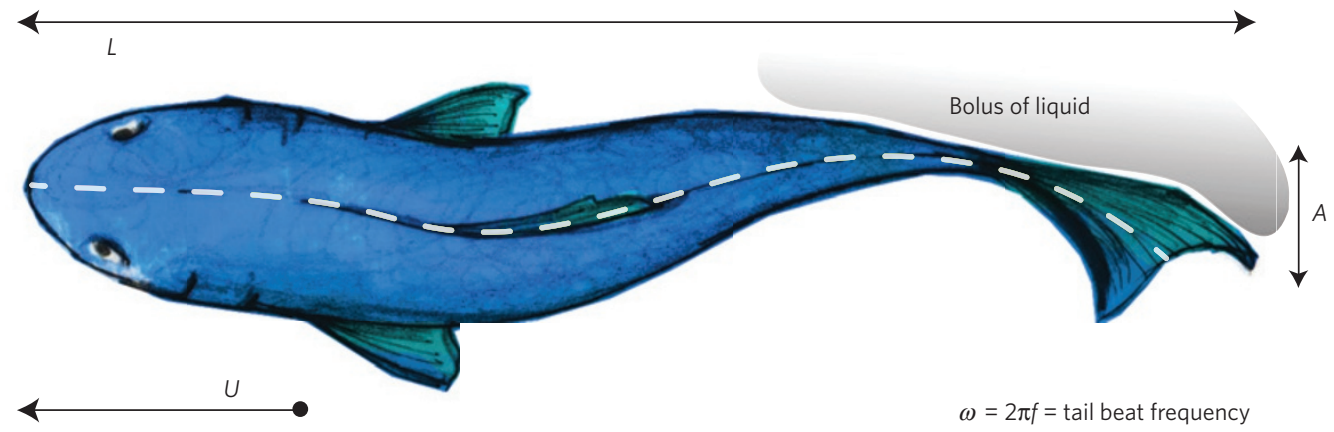

c

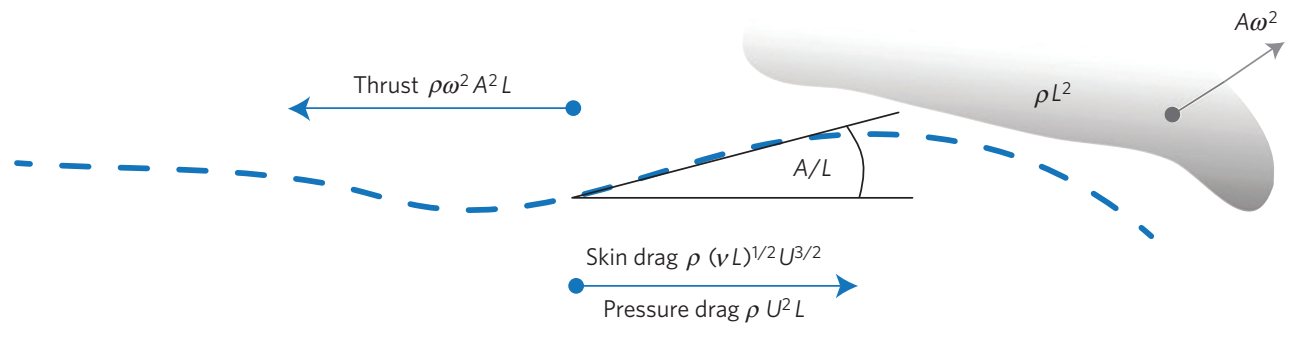

Figure 1 | Aquatic swimming. a, The organisms considered here (Supplementary Information) span eight orders of magnitude in Reynolds number and encompass larvae (from mayfly to zebrafish), fish (from goldfish, to stingrays and sharks), amphibians (tadpoles), reptiles (alligators), marine birds (penguins) and large mammals (from manatees and dolphins to belugas and blue whales). Blue fish sketch by Margherita Gazzola. b, Swimmer of length $L$ is propelled forward with velocity $U$ by pushing a bolus of water ${ }^{14,20,24}$ through body undulations characterized by tail beat amplitude $A$ and frequency $\omega$. c, Thrust and drag forces on a swimmer. Thrust is the reaction force associated with accelerating $\left(A \omega^{2}\right)$ the mass of liquid per unit depth $\rho L^{2}$ weighted by the local angle $A / L$ (therefore $\rho L A$ may be understood as the mass of liquid channelled downstream). For laminar boundary layers, the drag is dominated by viscous shear (skin drag), whereas for turbulent boundary layers, the drag is dominated by pressure (pressure drag).

As most species when swimming at high speeds maintain an approximately constant value of the specific tail beat amplitude $A / L$ (refs 8,11 ), relation (2) reduces to $U / L \sim f$, providing a mechanistic basis for Bainbridge's empirical relation.

In Fig. 2a, we plot all data from over 1,000 different measurements compiled from a variety of sources (Supplementary Information) in terms of $\mathrm{Re}$ and $\mathrm{Sw}$, for fish (from zebrafish larvae to stingrays and sharks), amphibians (tadpoles), reptiles (alligators), marine birds (penguins) and large mammals (from manatees and dolphins to belugas and blue whales). The organisms varied in size from 0.001 to $30 \mathrm{~m}$, while their propulsion frequency varied from 0.25 to $100 \mathrm{~Hz}$. The dimensionless numbers we use to scale the data provides a natural division of aquatic organisms by size, with fish larvae at the bottom left, followed by small amphibians, fish, birds, reptiles, and large marine mammals at the top right. We see that the data, which span nearly eight orders of magnitude in the Reynolds number, are in agreement with our predictions, and show a natural crossover from the laminar power law (1) to the turbulent power law (2) at a Reynolds number of approximately $\operatorname{Re} \simeq 3,000$. To understand this, we note that the skin friction starts to be dominated by the pressure drag when the thickness of the laminar boundary layer is comparable to half the oscillation amplitude. Therefore, a minimal estimate for the critical Reynolds number $\mathrm{Re}_{\text {critical }}$ associated with the laminarturbulent transition is given by the relation $\delta \simeq A / 2$. For a flat plate $^{26} \delta=5 \sqrt{\nu L / U}$ and given a typical value of $A / L=0.2$, we obtain $\operatorname{Re}_{\text {critical }} \simeq(10 L / A)^{2}=2,500$, which is in agreement with experimental data.

Naturally, some organisms do not hew exactly to our scaling relationships. Indeed, sirenians (manatees) slightly fall below the line, whereas anuran tadpoles lie slightly above it (Supplementary Information). We ascribe these differences to intermittent modes of locomotion involving a combination of acceleration, steady swimming and coasting that these species often use. Other reasons for the deviations could be related to different gaits in which part or the entire body is used, as in carangiform or anguilliform motion. Moreover, morphological variations associated with the body, tail and fins may play a role by directly affecting the hydrodynamic profile, or indirectly by modifying the gaits. However, the agreement with our minimal scaling arguments suggests that the role of these specifics is secondary, given the variety of shapes and gaits encompassed in our experimental data set. 
a

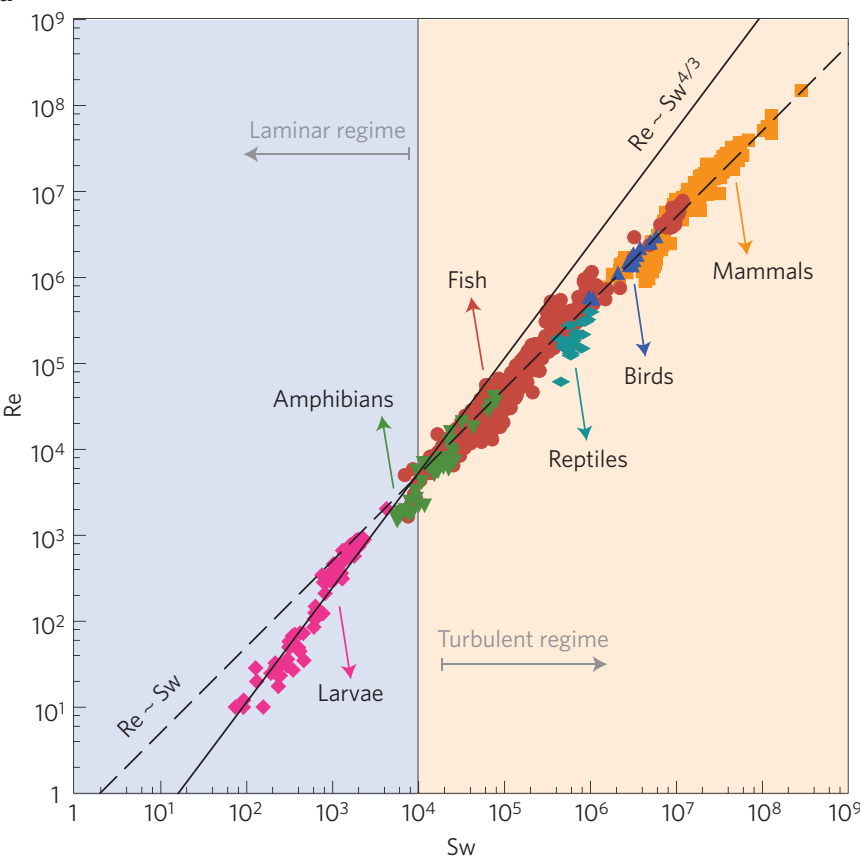

b

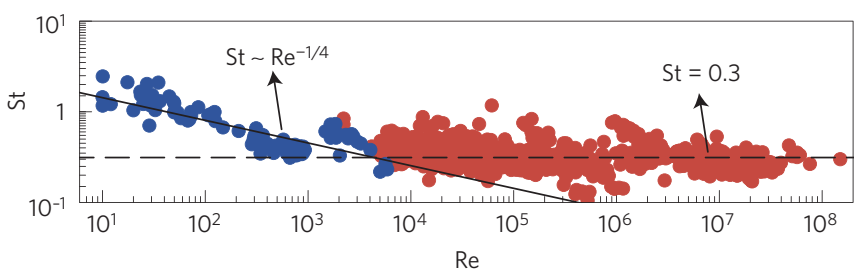

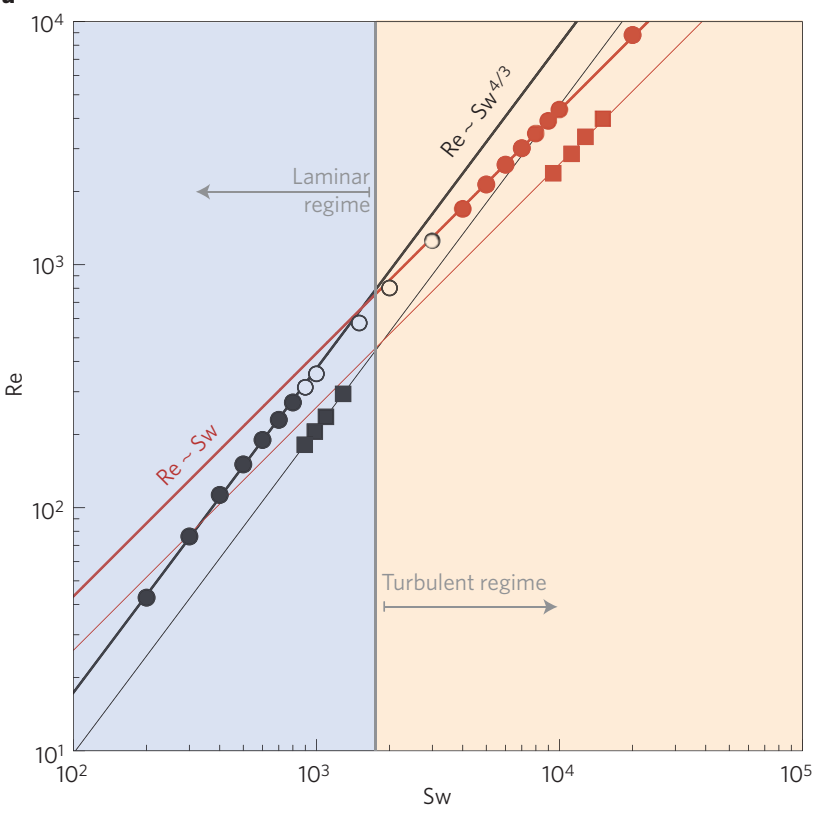
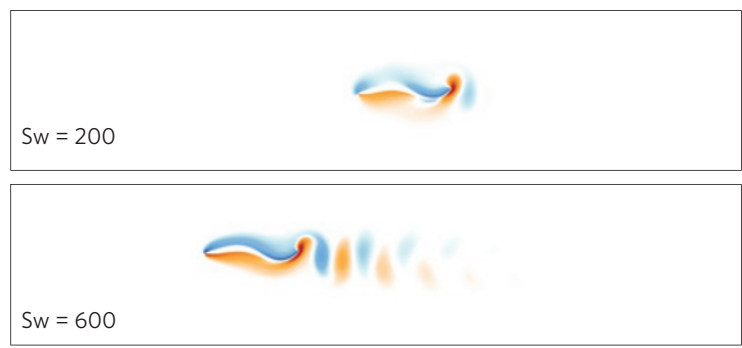

Figure 2 | Scaling aquatic locomotion: measurements. a, Data from amphibians, larvae, fish, marine birds and mammals show that the scaled speed of the organism $\operatorname{Re}=U L / v$ varies with the scaled frequency of the oscillatory propulsor $\mathrm{Sw}=\omega A L / v$ according to equations (1) and (2) over eight decades. Data fit for the laminar regime yields $\mathrm{Re}=0.03 \mathrm{Sw} \mathrm{w}^{1.31}$ with $R^{2}=0.95$, and for the turbulent regime yields $R e=0.4 S w^{1.02}$ with $R^{2}=0.99$. b. The Strouhal number St $=f A / U$, with $f=\omega / 2 \pi$, depends weakly on Reynolds number St $\sim \operatorname{Re}^{-1 / 4}$ for $\mathrm{Sw}<10^{4}$ (blue) and is independent for $\mathrm{Sw}>10^{4}$ (red), consistent with our scaling relationships and earlier observations ${ }^{30}$.

Because aquatic organisms live in water, testing the dependence of our scaling relationships on viscosity requires manipulating the environment. Although this has been done on occasion ${ }^{27}$ and is consistent with our scaling relations (Supplementary Information), numerical simulations of the Navier-Stokes equations coupled to the motion of a swimming body allow us to test our power laws directly by varying $S w$ via the viscosity $v$ only (Supplementary Information). In Fig. 3, we show the results for two-dimensional anguilliform swimmers ${ }^{28,29}$. The data from our numerical experiments straddle both sides of the crossover from the laminar to the turbulent regime and are in quantitative agreement with our minimal scaling theory, and our simple estimate for $\mathrm{Re}_{\text {critical }}$. To further challenge our theoretical scaling relationships, in Fig. 3, we plot the results of three-dimensional simulations performed by various groups using different numerical techniques ${ }^{19,22,24,28}$; they also collapse onto the same power laws (details in Supplementary Information). The agreement with both two- and three-dimensional numerical simulations, which are not affected by environmental and behavioural vagaries, gives us further confidence in our theory.

Traditionally, most studies of locomotion use the Strouhal number $\mathrm{St}=\omega A / U$, a variable borrowed from engineering, to

\section{Figure 3 | Scaling aquatic locomotion: simulations. a, Two- and} three-dimensional direct numerical simulations of swimming creatures confirm equations ( 1 ) and (2). Circles correspond to two-dimensional simulations, while squares correspond to three-dimensional simulations (details about sources and numerical techniques can be found in the Supplementary Information). In the case of two-dimensional simulations, a data fit for the laminar regime yields $R e=0.04 S w^{4 / 3}$ with $R^{2}=0.99$, and for the turbulent regime yields $R e=0.43 S$ w with $R^{2}=0.99$. Remarkably, three-dimensional simulations performed by various groups ${ }^{19,22,24,28}$ and with different numerical techniques (Supplementary Information) confirm our scaling relations $\left(\operatorname{Re}=0.02 \mathrm{Sw}^{4 / 3}\right.$ with $R^{2}=1.00$, and $\mathrm{Re}=0.26 \mathrm{~S}$ w with $R^{2}=0.99$ ). b. For several Sw we display the vorticity fields (red-positive, blue-negative) generated by a two-dimensional anguilliform swimmer initially located on the rightmost side of the figure.

characterize the underlying dynamics. Although this is reasonable for many engineering applications such as vortex shedding, vibration and so on, in a biological context it is worth emphasizing that St confounds input $A-\omega$ and output $U$ variables, captures only one length scale by assuming $A \sim L$, and does not account for varying fluid environments characterized by $v$. For biological locomotion, $\mathrm{Sw}$ is a more natural variable as it captures the 
two length scales associated with the tail amplitude and the body size, accounts for the fluid environment, and allows us to seamlessly relate the input kinematics $A-\omega$ to the output velocity $U$. Nevertheless, writing equations (1) and (2) in terms of St yields $\mathrm{Sw}=\mathrm{Re} \cdot \mathrm{St}$; therefore $\mathrm{St} \sim \mathrm{Re}^{-1 / 4}$, for laminar flows, and $\mathrm{St}=$ const, for turbulent flows, showing little or no influence of the Reynolds number on the Strouhal number. This direct consequence of our theory is consistent with experimental observations (Fig. 2b) and provides a physical basis for the findings ${ }^{30}$ that most swimming and flying animals operate in a relatively narrow range of St.

Despite the vast phylogenetic spread of inertial swimmers (Supplementary Information), we find that their locomotory dynamics is governed by the elementary hydrodynamical principles embodied in the power law $\operatorname{Re} \sim \mathrm{Sw}^{\alpha}$, with $\alpha=4 / 3$ for laminar regimes and $\alpha=1$ for turbulent boundary layers. This scaling relation follows by characterizing the biological diversity of aquatic locomotion in terms of the physical constraints of inertial swimming, a convergent evolutionary strategy for moving through water in macroscopic creatures. Recalling that the phase space $\mathrm{Sw}$-Re relates the input transverse Reynolds number Sw to the output longitudinal Reynolds number Re, our scaling relations might also be interpreted as the edge of optimal steady locomotor performance in this space, separating the inefficient (below) from the unattainable (above) steady regimes in oscillatory aquatic propulsive systems. We anticipate that if general principles for aerial or terrestrial locomotion exist, they may well be found by considering the physical limits dictated by their respective environments.

Received 10 April 2014; accepted 28 July 2014; published online 14 September 2014

\section{References}

1. Pedley, T. J. Scale Effects in Animal Locomotion (Cambridge Univ. Press, 1977).

2. Vogel, S. Life in Moving Fluids: The Physical Biology of Flow (Princeton Univ. Press, 1996).

3. Fish, F. E. \& Lauder, G. V. Passive and active flow control by swimming fishes and mammals. Annu. Rev. Fluid Mech. 38, 193-224 (2006).

4. Childress, S. Mechanics of Swimming and Flying (Cambridge Univ. Press, 1981).

5. Hertel, H. Structure, Form, Movement (Reinhold, 1966).

6. Triantafyllou, M. S., Triantafyllou, G. S. \& Yue, D. K. P. Hydrodynamics of fishlike swimming. Annu. Rev. Fluid Mech. 32, 33-53 (2000).

7. Wu, T. Y. Fish swimming and bird/insect flight. Annu. Rev. Fluid Mech. 43, 25-58 (2011).

8. Bainbridge, $\mathrm{R}$. The speed of swimming of fish as related to size and to the frequency and amplitude of the tail beat. J. Exp. Biol. 35, 109-133 (1958).

9. Webb, P. W., Kostecki, P. T. \& Stevens, E. D. The effect of size and swimming speed on locomotor kinematics of rainbow trout. J. Exp. Biol. 109, 77-95 (1984).

10. Videler, J. J. \& Wardle, C. S. Fish swimming stride by stride: speed limits and endurance. Rev. Fish Biol. Fisheries 1, 23-40 (1991).

11. Mchenry, M., Pell, C. \& Long, J. H. Jr Mechanical control of swimming speed: Stiffness and axial wave form in undulating fish models. J. Exp. Biol. 198, 2293-305 (1995).

12. Long, J. H. Jr Muscles, elastic energy, and the dynamics of body stiffness in swimming eels. Am. Zool. 38, 771-792 (1998).

13. Taylor, G. I. Analysis of the swimming of long and narrow animals. Proc. R. Soc. Lond. A 214, 158-183 (1952).
14. Lighthill, M. J. Large-amplitude elongated-body theory of fish locomotion. Proc. R. Soc. Lond. B 179, 125-138 (1971).

15. Hess, F. \& Videler, J. J. Fast continuous swimming of saithe (Pollachius virens): A dynamic analysis of bending moments and muscle power. J. Exp. Biol. 109, 229-251 (1984).

16. Cheng, J. Y., Pedley, T. J. \& Altringham, J. D. A continuous dynamic beam model for swimming fish. Phil. Trans. R. Soc. Lond. B 353, $981-997$ (1998).

17. McMillen, T., Williams, T. \& Holmes, P. Nonlinear muscles, passive viscoelasticity and body taper conspire to create neuromechanical phase lags in anguilliform swimmers. PLoS Comput. Biol. 4, e1000157 (2008).

18. Alben, S., Witt, C., Baker, T. V., Anderson, E. \& Lauder, G. V. Dynamics of freely swimming flexible foils. Phys. Fluids 24, 051901 (2012).

19. Kern, S. \& Koumoutsakos, P. Simulations of optimized anguilliform swimming. J. Exp. Biol. 209, 4841-4857 (2006).

20. Gazzola, M., van Rees, W. M. \& Koumoutsakos, P. C-start: Optimal start of larval fish. J. Fluid Mech. 698, 5-18 (2012).

21. Tytell, E. D., Hsu, C. Y., Williams, T. L., Cohen, A. H. \& Fauci, L. J. Interactions between internal forces, body stiffness, and fluid environment in a neuromechanical model of lamprey swimming. Proc. Natl Acad. Sci. USA 107, 19832-19837 (2010).

22. Tytell, E. D. et al. Disentangling the functional roles of morphology and motion in the swimming of fish. Integr. Comp. Biol. 50, 1140-1154 (2010).

23. Bhalla, A. P. S., Griffith, B. E. \& Patankar, N. A. A forced damped oscillation framework for undulatory swimming provides new insights into how propulsion arises in active and passive swimming. PLoS Comput. Biol. 9, e1003097 (2013).

24. van Rees, W. M., Gazzola, M. \& Koumoutsakos, P. Optimal shapes for anguilliform swimmers at intermediate Reynolds numbers. J. Fluid Mech. 722, R3 (2013).

25. Batchelor, G. K. An Introduction to Fluid Dynamics (Cambridge Univ. Press, 1967).

26. Landau, L. D. \& Lifshitz, E. M. Fluid Mechanics (Pergamon Press, 1959).

27. Horner, A. M. \& Jayne, B. C. The effects of viscosity on the axial motor pattern and kinematics of the African lungfish (Protopterus annectens) during lateral undulatory swimming. J. Exp. Biol. 211, 1612-1622 (2008).

28. Gazzola, M., Chatelain, P., van Rees, W. M. \& Koumoutsakos, P. Simulations of single and multiple swimmers with non-divergence free deforming geometries. J. Comput. Phys. 230, 7093-7114 (2011).

29. Gazzola, M., Hejazialhosseini, B. \& Koumoutsakos, P. Reinforcement learning and wavelet adapted vortex methods for simulations of self-propelled swimmers. SIAM J. Sci. Comput. 36, B622-B639 (2014).

30. Taylor, G. K., Nudds, R. L. \& Thomas, A. L. R. Flying and swimming animals cruise at a Strouhal number tuned for high power efficiency. Nature 425, 707-711 (2003).

\section{Acknowledgements}

We thank the Swiss National Science Foundation, and the MacArthur Foundation for partial financial support. We also thank W. van Rees, B. Hejazialhosseini and P. Koumoutsakos of the CSElab at ETH Zurich for their help with the computational aspects of the study, and Margherita Gazzola for her sketches.

\section{Author contributions}

M.G., M.A. and L.M. conceived the study, developed the theory, collected and analysed the experimental data and wrote the paper. M.G. performed the $2 \mathrm{D}$ numerical simulations.

\section{Additional information}

Supplementary information is available in the online version of the paper. Reprints and permissions information is available online at www.nature.com/reprints.

Correspondence and requests for materials should be addressed to L.M.

\section{Competing financial interests}

The authors declare no competing financial interests. 